\title{
Vibration and sound radiation of a rotating train wheel subject to a vertical harmonic wheel-rail force
}

\author{
Tingsheng Zhong ${ }^{1} \cdot$ Gong Chen $^{1} \cdot$ Xiaozhen Sheng $^{1}$ (1) $\cdot$ Xueyan Zhan $^{2} \cdot$ \\ Liqun $\mathrm{Zhou}^{1} \cdot$ Jian Kai $^{1}$
}

Received: 26 September 2017/Revised: 5 December 2017/Accepted: 8 December 2017/Published online: 8 January 2018

(C) The Author(s) 2018. This article is an open access publication

\begin{abstract}
The rapid development of high-speed railway networks requires advanced methods for analysing vibration and sound radiation characteristics of a fast rotating train wheel subject to a vertical harmonic wheel-rail force. In order to consider the rotation of the wheel and at the same time increase the computational efficiency, a procedure is adapted in this paper taking advantage of the axial symmetry of the wheel. In this procedure, a recently developed 2.5D finite element method, which can consider wheel rotation but only requires a $2 \mathrm{D}$ mesh over a cross section containing the wheel axis, is used to calculate the vibration response of the wheel. Then, the vibration response of the wheel is taken as acoustic boundary condition and the 2.5D acoustic boundary element method, which only requires a 1D mesh over the boundary of the above cross section, is utilised to calculate the sound radiation of the wheel. These $2.5 \mathrm{D}$ methods and relevant programs are validated by comparing results from this procedure with those from conventional 3D analyses using commercial software. The comparison also demonstrates that these $2.5 \mathrm{D}$ methods have a much higher computational efficiency. Using the $2.5 \mathrm{D}$ methods, we study the wheel rotation speed influences on the factors including the vertical receptance of the wheel at wheel-rail contact point, sound pressure level at a pre-defined standard measurement point, radiated sound power level, directivity of the radiation, and contribution of each part of the wheel. It can be
\end{abstract}

Xiaozhen Sheng

shengxiaozhen@hotmail.com

1 State Key Laboratory of Traction Power, Southwest Jiaotong University, Chengdu 610031, China

2 Faculty of Geosciences and Environmental Engineering, Southwest Jiaotong University, Chengdu 610031, China concluded that the wheel rotation speed splits most peaks of the vertical receptance at the wheel-rail contact point, sound pressure levels at the field, and the sound power level of the wheel into two peaks. The directivity and power contribution of the wheel are also significantly changed by the wheel rotation speed. Therefore, the rotation of a train wheel should be taken into account when calculating its vibration and sound radiation.

Keywords Train wheel · Vibration response - Sound radiation $\cdot$ 2.5D FEM $\cdot 2.5 \mathrm{D} \mathrm{BEM} \cdot$ Rotation effect

\section{Introduction}

With the rapid development of high-speed railway networks, noise pollution from railways is becoming more and more serious. Wheel/rail noise and aerodynamic noise are main noise sources of high-speed trains. Wheel/rail noise mainly comes from sounds radiated from wheel and rail vibrations [1]. According to Thompson [2], for a typical ballasted track, noise from wheel radiation is dominant in wheel/rail noise for frequencies higher than $2500 \mathrm{~Hz}$. Therefore, wheel vibro-acoustic analysis is an important topic.

Many researchers have studied the vibration and sound radiation characteristics of a train wheel. The wheel was greatly simplified in early work. In Refs. [3, 4], to calculate wheel vibration response, the wheel was assumed to be a rigid mass and a point noise source for estimating sound radiation. However, in such a simplified model, valid frequency range is very limited. Therefore, Remington, in subsequent work [5, 6], used the Love's ring theory to build a wheel model in which the wheel tyre was regarded as an elastic thin ring, the web was simplified as springs 
and dampers, and the hub combined with the axle was taken to be a rigid mass. A relationship between the radiation efficiency of the wheel and frequency was obtained by fitting experimental data. However, this model is still limited, as it is not applicable to calculating noise radiations from vibrations of the wheel web.

Numerical modelling has been increasingly used with the advancement in computing capability. For example, Schneider [7] used the ring element to build a finite element model for a train wheel and calculated sound radiation of the wheel using the Rayleigh integral method. Thompson used a two-dimensional finite element model to analyse the free vibration characteristics of a train wheel in detail [8] and the two-dimensional boundary element method to analyse sound radiation. In another work [9], he studied the influences of wheel diameter, web, and hub thickness on sound radiation efficiency and directivity of the wheel and produced formulae for sound radiation efficiency via curve fitting.

Now three-dimensional finite and/or boundary element methods are generally used to perform vibro-acoustics for a train wheel. Sato et al. [10] conducted experimental tests to investigate the vibration and sound radiation characteristics of a wheel either with a wavy web or with a straight web. They combined with the finite element method and boundary element method to study sound radiation characteristics of different parts of the wheel, including contributions of the parts to the overall sound radiation of the wheel. The vibration response and sound radiation of a wheel subject to a vertical irregular excitation were simulated, and the influence of train speed on the vibration response and sound radiation of the wheel was discussed by Fang et al. [11], taking the wheel-rail contact filter effect into consideration.

Although there are a plenty of researches on vibration response and sound radiation of a train wheel, the effects of wheel rotation have not been adequately addressed. It has recently demonstrated in Ref. [12] that, at high speeds the rotation of a wheel has a significant effect on wheel dynamics. From this, it would be expected that the rotation of the wheel may also have an important effect on sound radiation. In other words, for a train wheel moving at high speeds, wheel rotation should be considered when performing vibro-acoustic analyses for the wheel.

As stated above, the traditional 3D BEM (in time domain and/or frequency domain) is commonly used to calculate sound radiations from a train wheel. However, the calculation time is too long for a fast rotating wheel vibrating at high frequencies. Thus, a procedure is required to be able to take wheel rotation into account and have higher computational efficiency.

A train wheel is a moving and rotating noise source if observed from the ground, but it is only a rotating noise source if observed from the train. In this paper, sound radiation of a rotating train wheel is studied only from the train. To develop the desirable procedure in the case of the high-speed rotation of the wheel, the vibration response of the rotating wheel calculated by the 2.5D FEM in Ref. [12] is taken as an acoustic boundary condition. In order to improve the calculation efficiency of wheel sound radiation, the axial symmetry of the wheel is utilised.

Many scholars have studied the application of boundary element method in fast calculation of sound radiation of axisymmetric body. Seybert et al. [13] proposed an approach in which the surface Helmholtz integral was reduced to a line integral and an integral over the angle revolution. The second integral was evaluated partly analytically in terms of elliptic integrals and partly numerically using Gaussian quadrature. The boundary element method was then used to perform the line integration. Soenarko [14] used Fourier series expansion to simplify the three-dimensional acoustic integral equation to a 1D integral along the profile curve of the body, and for each Fourier term used the boundary element method to realise sound radiation calculations for given boundary conditions (Fourier acoustic boundary element method, or 2.5D BEM). Kuijpers et al. [15] used similar approach incorporated with the fast Fourier transform (FFT) technique. It should be pointed out that no rotation occurs for the axisymmetric body considered in those 2.5D acoustic BEM work. How to incorporate wheel rotation into the $2.5 \mathrm{D}$ acoustic BEM is a topic of the current paper.

In this paper, the above-referred 2.5D FEM and 2.5D BEM are combined to calculate vibration and sound radiation of a wheel (with or without rotation) subject to a unit vertical harmonic wheel-rail force. Vibration and sound radiation of the wheel calculated by this method are compared with those calculated by commercial software to verify the method derivation and related programs. Finally, the influences of the wheel rotation speed on the wheel vibration response and sound radiation are discussed.

This paper is organised as follows. The 2.5D FEM used to calculate wheel vibration characteristics is briefly introduced in Sect. 2. The 2.5D BEM used to calculate wheel sound radiation characteristics is presented in Sect. 3. Results for a typical high-speed train wheel are presented in Sect. 4. Finally, conclusions are summarised in Sect. 5.

\section{2.5D FEM for calculating train wheel vibration}

This section briefly describes the 2.5D FEM for calculating the vibration response of a stationary or rotating wheel subject to a vertical harmonic wheel-rail force. Detailed derivations of the method can be found in Ref. [12]. 


\subsection{Differential equations of motion of the wheel}

As shown in Fig. 1, coordinate system oxyz is rigidly attached on the wheel with the $y$-axis coinciding with the wheel axis. It rotates uniformly about the $y$-axis at the wheel rotation speed $\Omega_{y}$ in the direction shown. ( $\Omega_{y}$ is equal to the train speed divided by the rolling radius of the wheel.) Observed from an inertial frame of reference, $O X Y Z$, which moves uniformly with the train in the track direction, the $y$-axis is allowed to vibrate in the vertical direction only.

According to the Lagrange's equation of the second kind, the differential equation of motion of an element volume is given by

$$
\begin{gathered}
\boldsymbol{M} \ddot{\boldsymbol{q}}(\theta, t)+\boldsymbol{K}_{0} \boldsymbol{q}(\theta, t)+\boldsymbol{R}_{1} \frac{\partial \boldsymbol{q}(\theta, t)}{\partial \theta} \\
=\boldsymbol{f}_{0}(\theta, t)+\boldsymbol{f}_{1}(\theta, t)+\boldsymbol{f}_{2}(\theta, t)
\end{gathered}
$$

where $\boldsymbol{q}(\theta, t)$ is the nodal displacement vector of the element volume observed from oxyz; $\boldsymbol{M}, \boldsymbol{K}_{0}$, and $\boldsymbol{R}_{1}$ are $3 n \times 3 n$ matrices (where, $n$ is the number of nodes of the element volume); $\boldsymbol{f}_{0}(\theta, t)$ is a nodal force vector associated with externally applied loads; $\boldsymbol{f}_{1}(\theta, t)$ is a nodal force vector associated with stresses on the $\theta$ and $\theta+\mathrm{d} \theta$ cross sections of the element volume; and $\boldsymbol{f}_{2}(\theta, t)$ is a nodal force vector associated with the motion of the oxyz system. $\boldsymbol{f}_{1}(\theta, t)$ can be obtained through the virtual work done by the stresses on the $\theta$ and $\theta+\mathrm{d} \theta$ cross sections of the element volume, and $\boldsymbol{f}_{2}(\theta, t)$ can be obtained through the virtual work done by the convection inertial forces of the element volume. With the last two nodal force vectors submitted, Eq. (1) becomes

$$
\begin{gathered}
\boldsymbol{M} \ddot{\boldsymbol{q}}(\theta, t)-2 \Omega_{y} \boldsymbol{G} \dot{\boldsymbol{q}}(\theta, t)+\left(\boldsymbol{K}_{0}-\Omega_{y}^{2} \boldsymbol{M}_{\mathrm{c}}\right) \boldsymbol{q}(\theta, t) \\
\quad+\boldsymbol{K}_{1} \frac{\partial q(\theta, t)}{\partial \theta}-\boldsymbol{K}_{2} \frac{\partial^{2} \boldsymbol{q}(\theta, t)}{\partial \theta^{2}} \\
=\boldsymbol{f}_{0}(\theta, t)+\Omega_{y}^{2} \boldsymbol{h}+\boldsymbol{h}_{1} \ddot{w}_{0}(t) \sin \left(\Omega_{y} t+\theta\right) \\
\quad+\boldsymbol{h}_{2} \ddot{w}_{0}(t) \cos \left(\Omega_{y} t+\theta\right),
\end{gathered}
$$

where $\boldsymbol{M}$ is mass matrix; $\boldsymbol{K}_{0}$ is stiffness matrix; and $\boldsymbol{K}_{2}$ and $\boldsymbol{M}_{\mathrm{c}}$ are stiffness-like matrices, all symmetric; $\boldsymbol{K}_{1}$ and $\boldsymbol{G}$ are anti-symmetric matrices; $\boldsymbol{G}$ and $\boldsymbol{M}_{\mathrm{c}}$ are associated with the gyroscopic (via the term $-2 \Omega_{y} \boldsymbol{G} \dot{\boldsymbol{q}}(\theta, t)$ ) and centrifugal (via the term $-\Omega_{y}^{2} \boldsymbol{M}_{\mathrm{c}} \boldsymbol{q}(\theta, t)$ ) effects induced by wheel rotation; $\boldsymbol{h}, \boldsymbol{h}_{1}$, and $\boldsymbol{h}_{2}$ are vectors. $\Omega_{y}^{2} \boldsymbol{h}$ is the static centrifugal force vector which causes no vibration to the wheel; therefore, this term will be dropped hereinafter.

Equation (2) is for an element on the $\theta$-plane. (Internal forces between elements are not included, since they disappear in the global FE equation.) Similar equation can be established for each and every element on the $\theta$-plane. The conventional finite element 'summation' of the element matrices in Eq. (2) is used to obtain the corresponding matrices of the assembled finite element model and thus the global differential equation of motion. This is still represented by Eq. (2). In other words, $\boldsymbol{q}(\theta, t)$ now is a vector containing all the degrees of freedom on the $\theta$-plane.

It can be seen from Eq. (2) that the FE equation contains the vertical acceleration, $\ddot{w}_{0}(t)$ of the mass centre of the wheel, and this is also to be determined. Therefore, an extra equation is required. This extra equation may be established by applying the momentum law to the wheel in the vertical direction. This gives

$$
\begin{aligned}
\sum F_{Z}(t)= & m_{W} \ddot{w}_{0}(t)-\Omega_{y}^{2} \int_{0}^{2 \pi}\left(\sin \left(\Omega_{y} t+\theta\right), 0,\right. \\
& \left.\times \cos \left(\Omega_{y} t+\theta\right)\right) \boldsymbol{S} \boldsymbol{q}(\theta, t) \mathrm{d} \theta \\
& +2 \Omega_{y} \int_{0}^{2 \pi}\left(\cos \left(\Omega_{y} t+\theta\right), 0,-\sin \left(\Omega_{y} t+\theta\right)\right) \boldsymbol{S} \dot{\boldsymbol{q}}(\theta, t) \mathrm{d} \theta \\
& +\int_{0}^{2 \pi}\left(\sin \left(\Omega_{y} t+\theta\right), 0, \cos \left(\Omega_{y} t+\theta\right)\right) \boldsymbol{S} \ddot{\boldsymbol{q}}(\theta, t) \mathrm{d} \theta,
\end{aligned}
$$

where $m_{W}$ denotes the total mass of the wheel, and $S$ is a $3 \times N$ matrix (where $N$ is the total dofs on the $\theta$-plane).

\subsection{Response of a rotating wheel to a vertical harmonic wheel-rail force}

For rolling noise generation, the vertical wheel/rail force component is the most important [8]. Observed from the wheel, a vertical wheel-rail force is rotating along the wheel rolling surface. In terms of the model presented in Sect. 2.1, the wheel-rail force can be described by (see Fig. 1)

$\boldsymbol{f}_{0}(\theta, t)=\boldsymbol{p}_{0} \delta\left(\theta+\Omega_{y} t-\pi / 2\right) \mathrm{e}^{\mathrm{i} \Omega t}$,

where $\boldsymbol{p}_{0}$ is a vector. Since $\boldsymbol{q}(\theta, t)$ is a periodic function of $\theta$ with the period being $2 \pi$, it can be expressed as a Fourier series,

$\boldsymbol{q}(\theta, t)=\sum_{m=-\infty}^{\infty} \tilde{\boldsymbol{q}}_{m}(t) \mathrm{e}^{\mathrm{i} m \theta}$,

where $m$ is a circumferential order.

After a series of derivations, we can obtain 


$$
\left.\begin{array}{rl}
\boldsymbol{M} \ddot{\tilde{\boldsymbol{q}}}_{m}(t)-2 \Omega_{y} \boldsymbol{G} \dot{\tilde{\boldsymbol{q}}}_{m}(t)+\left(\boldsymbol{K}_{0}-\Omega_{y}^{2} \boldsymbol{M}_{\mathrm{c}}\right) \tilde{\boldsymbol{q}}_{m}(t)+\mathrm{i} m \boldsymbol{K}_{1} \tilde{\boldsymbol{q}}_{m}(t)+m^{2} \boldsymbol{K}_{2} \tilde{\boldsymbol{q}}_{m}(t)=\frac{1}{2 \pi} \boldsymbol{p}_{0} \mathrm{e}^{-\mathrm{i} m \pi / 2} \mathrm{e}^{\mathrm{i}\left(\Omega+m \Omega_{y}\right) t},(m \neq \pm 1) \\
\boldsymbol{M} \ddot{\tilde{\boldsymbol{q}}}_{-1}(t)-2 \Omega_{y} \boldsymbol{G} \dot{\tilde{\boldsymbol{q}}}_{-1}(t)+\left(\boldsymbol{K}_{0}-\Omega_{y}^{2} \boldsymbol{M}_{\mathrm{c}}\right) \tilde{\boldsymbol{q}}_{-1}(t)-\mathrm{i} \boldsymbol{K}_{1} \tilde{\boldsymbol{q}}_{-1}(t)+\boldsymbol{K}_{2} \tilde{\boldsymbol{q}}_{-1}(t) \\
=\frac{\mathrm{i}}{2 \pi} \boldsymbol{p}_{0} \mathrm{e}^{\mathrm{i}\left(\Omega-\Omega_{y}\right) t}+\frac{\mathrm{i}}{2} \boldsymbol{h}_{1} \ddot{w}_{0}(t) \mathrm{e}^{-\mathrm{i} \Omega_{y} t}+\frac{1}{2} \boldsymbol{h}_{2} \ddot{w}_{0}(t) \mathrm{e}^{-\mathrm{i} \Omega_{y} t} \\
\boldsymbol{M} \ddot{\tilde{\boldsymbol{q}}}_{1}(t)-2 \Omega_{y} \boldsymbol{G} \dot{\tilde{\boldsymbol{q}}}_{1}(t)+\left(\boldsymbol{K}_{0}-\Omega_{y}^{2} \boldsymbol{M}_{\mathrm{c}}\right) \tilde{\boldsymbol{q}}_{1}(t)+\mathrm{i} \boldsymbol{K}_{1} \tilde{\boldsymbol{q}}_{1}(t)+\boldsymbol{K}_{2} \tilde{\boldsymbol{q}}_{-1}(t) \\
=\frac{-\mathrm{i}}{2 \pi} \boldsymbol{p}_{0} \mathrm{e}^{\mathrm{i}\left(\Omega+\Omega_{y}\right) t}-\frac{\mathrm{i}}{2} \boldsymbol{h}_{1} \ddot{w}_{0}(t) \mathrm{e}^{\mathrm{i} \Omega_{y} t}+\frac{1}{2} \boldsymbol{h}_{2} \ddot{w}_{0}(t) \mathrm{e}^{\mathrm{i} \Omega_{y} t} \\
m_{W} \ddot{w}_{0}(t)-\Omega_{y}^{2}(\mathrm{i} \pi, 0, \pi) \boldsymbol{S} \tilde{\boldsymbol{q}}_{1}(t) \mathrm{e}^{-\mathrm{i} \Omega_{y} t}-\Omega_{y}^{2}(-\mathrm{i} \pi, 0, \pi) \boldsymbol{S} \tilde{\boldsymbol{q}}_{-1}(t) \mathrm{e}^{\mathrm{i} \Omega_{y} t}+2 \Omega_{y}(\pi, 0,-\mathrm{i} \pi) \boldsymbol{S} \dot{\tilde{\boldsymbol{q}}}_{1}(t) \mathrm{e}^{-\mathrm{i} \Omega_{y} t} \\
+2 \Omega_{y}(\pi, 0, \mathrm{i} \pi) \boldsymbol{S} \dot{\tilde{\boldsymbol{q}}}_{-1}(t) \mathrm{e}^{\mathrm{i} \Omega_{y} t}+(\mathrm{i} \pi, 0, \pi) \boldsymbol{S} \ddot{\tilde{\boldsymbol{q}}}_{1}(t) \mathrm{e}^{-\mathrm{i} \Omega_{y} t}+(-\mathrm{i} \pi, 0, \pi) \boldsymbol{S} \ddot{\tilde{q}}_{-1}(t) \mathrm{e}^{\mathrm{i} \Omega_{y} t}=-P_{0} \mathrm{e}^{\mathrm{i} \Omega_{y} t}
\end{array}\right\}
$$

By letting

$\tilde{\boldsymbol{q}}_{m}(t)=\hat{\boldsymbol{q}}_{m} \mathrm{e}^{\mathrm{i}\left(\Omega+m \Omega_{y}\right) t}, w_{0}(t)=\hat{w}_{0} \mathrm{e}^{\mathrm{i} \Omega t}$,

in Eq. (6), a set of linear algebraic equations is yielded which governs $\hat{\boldsymbol{q}}_{m}$ and $\hat{w}_{0}$.

It can be shown that, observed from $O X Y Z$, the vertical displacement of the wheel at the wheel-rail contact point is given by

$W(t)=\left(\hat{w}_{0}+\sum_{m=-\infty}^{\infty} \hat{\varphi}_{m} \mathrm{e}^{\mathrm{i} m \frac{\pi}{2}}\right) \mathrm{e}^{\mathrm{i} \Omega t}$,

where $\hat{\varphi}_{m}$ denotes the component in $\hat{\boldsymbol{q}}_{m}$ corresponding to the radial displacement of the wheel at the wheel-rail contact point. Equation (8) shows that the vertical vibration at the wheel-rail contact point is harmonic at the same frequency as the wheel-rail force. Thus, vertical receptance can be defined for the wheel at the wheel-rail contact point by setting the vertical wheel-rail force to be unit.

\subsection{Particle vibration velocity of air on the surface in touch with the wheel}

Since sound radiation from the wheel is observed from the train (i.e. from $O X Y Z$ ), the sound field generated by the wheel is solely determined by the normal vibrational velocity seen from air on the boundary of the wheel even though the wheel is also in rotation. It can be shown that, observed from $O X Y Z$, the radial displacement vector of a cross section boundary of the wheel is given by (see Fig. 2 . This cross section is fixed if observed from air but moving if observed from the wheel)

$$
\begin{aligned}
\boldsymbol{Q}_{r}(\alpha, t) & =\boldsymbol{q}_{r}(t)+w_{0}(t) \sin \alpha \\
& =\left(\sum_{m=-\infty}^{\infty} \hat{\boldsymbol{q}}_{r m} \mathrm{e}^{\mathrm{i} m \alpha}+\hat{w}_{0} \sin \alpha\right) \mathrm{e}^{\mathrm{i} \Omega t}=\hat{\boldsymbol{Q}}_{r}(\alpha) \mathrm{e}^{\mathrm{i} \Omega t},
\end{aligned}
$$

where $\boldsymbol{q}_{r}(t)$ denotes the component in $\boldsymbol{q}(\theta, t)$ corresponding to the radial displacements of the cross section boundary of the wheel, and $\alpha$ denotes the angle of the cross section measured from the $X Y$ plane. Equation (9) shows that the radial vibration of the cross section boundary of the wheel is harmonic at the same frequency as the wheel-rail force, and its vibration displacement amplitude is periodic function of $\alpha$. It can also be shown that the axial and circumferential vibration of the cross section boundary is also harmonic at the same frequency as the wheel-rail force and their vibration displacements are periodic function of $\alpha$. Therefore, vibration velocity of air particle is harmonic at the same frequency as the wheel-rail force, and on the surface in touch with the wheel, it is a periodic function of $\alpha$ with period being $2 \pi$.

Since $\hat{\boldsymbol{Q}}_{r}(\alpha)$ is periodic function of $\alpha$ with the period of $2 \pi$, it can be expressed as a Fourier series,

$\hat{\boldsymbol{Q}}_{r}(\alpha)=\sum_{m=-\infty}^{\infty} \overline{\boldsymbol{Q}}_{r m} \mathrm{e}^{\mathrm{i} m \alpha}$,

where according to Eq. (9),

$$
\begin{aligned}
\overline{\boldsymbol{Q}}_{r m} & =\frac{1}{2 \pi} \int_{0}^{2 \pi} \hat{\boldsymbol{Q}}_{r}(\alpha) \mathrm{e}^{-\mathrm{i} m^{\prime} \alpha} \mathrm{d} \alpha \\
& =\hat{\boldsymbol{q}}_{r m}+\frac{\hat{w}_{0}}{2 \pi} \int_{0}^{2 \pi} \frac{\mathrm{e}^{\mathrm{i} \alpha}-\mathrm{e}^{-\mathrm{i} \alpha}}{2 i} \mathrm{e}^{-\mathrm{i} m \alpha} \mathrm{d} \alpha \\
& =\left\{\begin{array}{l}
\hat{\boldsymbol{q}}_{r m}-\frac{\hat{w}_{0}}{2 i}, m=-1 \\
\hat{\boldsymbol{q}}_{r m}+\frac{\hat{w}_{0}}{2 i}, m=1 \\
\hat{\boldsymbol{q}}_{r m}, \quad m \neq \pm 1
\end{array}\right.
\end{aligned}
$$

Therefore, the nodal normal vibration velocity vector of the cross section boundary of the wheel, shown in Fig. 2, corresponding to the circumferential order $m$ is

$\overline{\boldsymbol{v}}_{n m}=\mathrm{i} \Omega\left(\overline{\boldsymbol{q}}_{y m} n_{y}+\overline{\boldsymbol{Q}}_{r m} n_{r}\right)$,

where $\overline{\boldsymbol{q}}_{y m}$ denotes the component in $\hat{\boldsymbol{q}}_{m}$ corresponding to the axial nodal displacements of the cross section 


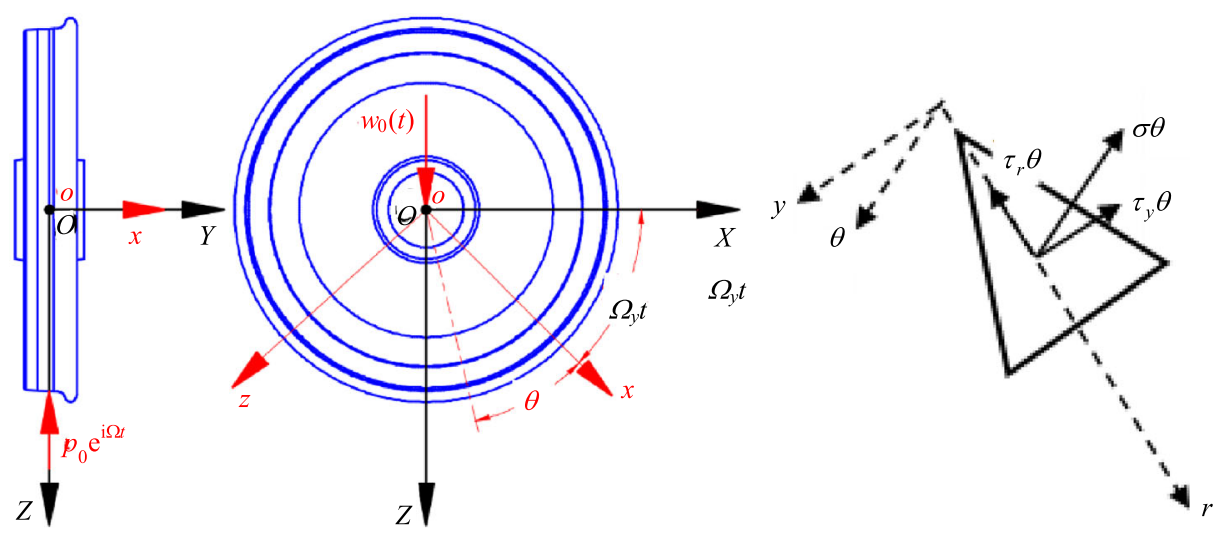

Fig. 1 Coordinate systems and an element volume

boundary, $\boldsymbol{n}_{r}$ and $\boldsymbol{n}_{y}$ are diagonal matrices consisting of the directional cosines of the normal vector in the radial and axial directions of the cross section boundary. The normal of the cross section boundary is directed into the wheel. With the boundary condition determined in Eq. (12), sound radiations from the wheel can be predicted as if the wheel were not in rotation.

\section{2.5D BEM for calculating train wheel sound radiation}

\subsection{5D boundary element equation}

Figure 3 shows a schematic diagram of the acoustic structure of a train wheel. The harmonic vibration and sound radiation of such structures can be simplified by their axial symmetry. In terms of the cylindrical coordinates, the acoustic boundary integral equation can be written as $[16,17]$

$$
\begin{aligned}
& C(r, y, \theta) \tilde{p}(r, y, \theta) \\
& =-\int_{0}^{2 \pi} \int_{\Gamma}\left[\tilde{p}\left(r^{\prime}, y^{\prime}, \theta^{\prime}\right) \frac{\partial G\left(r^{\prime}, y^{\prime}, \theta^{\prime} ; r, y, \theta\right)}{\partial n}\right. \\
& \left.\quad+\mathrm{i} \rho \omega G\left(r^{\prime}, y^{\prime}, \theta^{\prime} ; r, y, \theta\right) \tilde{v}_{n}\left(r^{\prime}, y^{\prime}, \theta^{\prime}\right)\right] r^{\prime} \mathrm{d} \Gamma \mathrm{d} \theta^{\prime},
\end{aligned}
$$

where $\tilde{p}(r, y, \theta)$ denotes the sound pressure amplitude at $(r, y, \theta)$ in the acoustic analysis field outside the wheel; $\tilde{p}\left(r^{\prime}, y^{\prime}, \theta^{\prime}\right)$ and $\tilde{v}_{n}\left(r^{\prime}, y^{\prime}, \theta^{\prime}\right)$ denotes the sound pressure amplitude and the normal vibration velocity amplitude at $\left(r^{\prime}, y^{\prime}, \theta^{\prime}\right)$ on the wheel surface, respectively; $G\left(r^{\prime}, y^{\prime}, \theta^{\prime} ; r, y, \theta\right)$ is the Green function, with source at $\left(r^{\prime}, y^{\prime}, \theta^{\prime}\right)$ and observer at $(r, y, \theta) ; C(r, y, \theta)$ denotes coefficient; $\Gamma$ denotes the cross section boundary of the wheel; $\boldsymbol{n}$ denotes the unit normal vector at source point on the

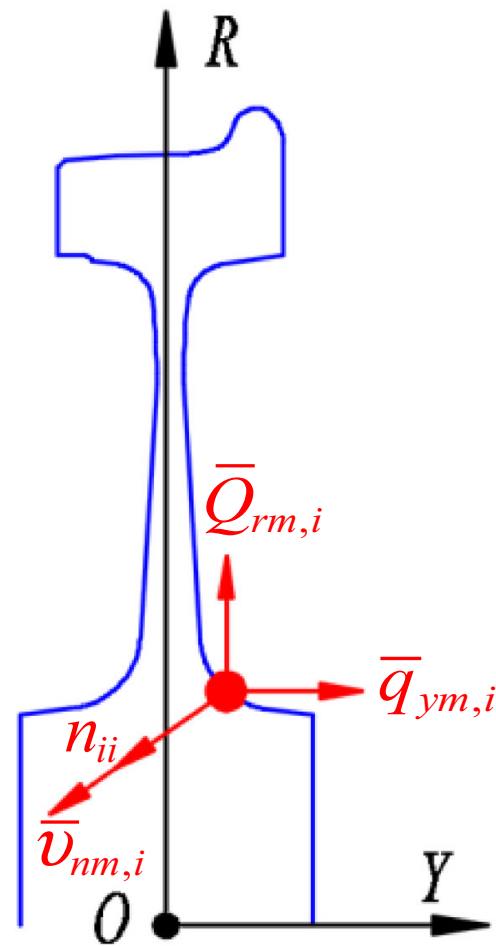

Fig. 2 Normal vibration velocity

wheel surface (directed into the wheel); $\rho$ denotes air density.

In terms of the Cartesian coordinates, the Green function is given by [17]

$$
\begin{aligned}
G\left(x^{\prime}, y^{\prime}, z^{\prime} ; x, y, z\right) & =\frac{\mathrm{e}^{-\mathrm{i} k R}}{4 \pi R}, k=\frac{\omega}{c}, R \\
& =\sqrt{\left(x^{\prime}-x\right)^{2}+\left(y^{\prime}-y\right)^{2}+\left(z^{\prime}-z\right)^{2}},
\end{aligned}
$$

The value of $C(r, y, \theta)$ is given by [18] 


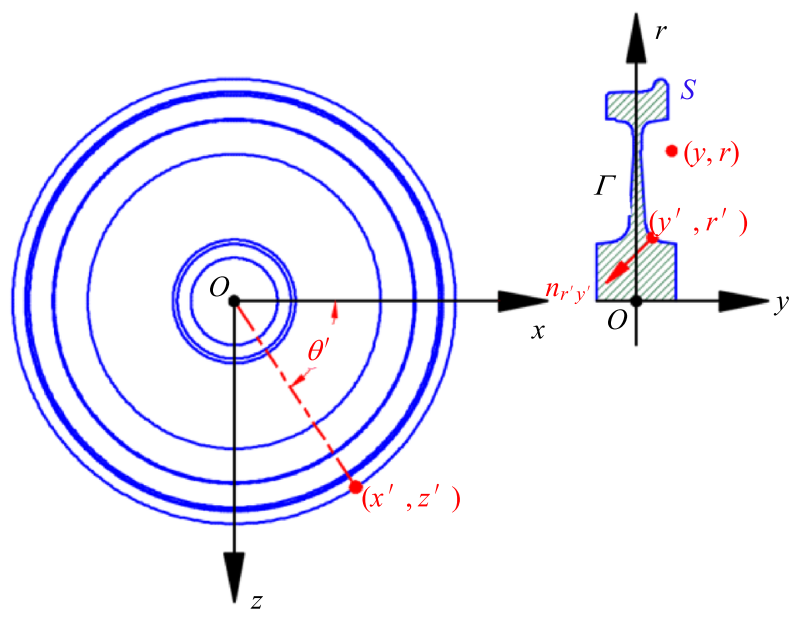

Fig. 3 Acoustic structure of a train wheel

$C(r, y, \theta)=\left\{\begin{array}{l}1,(r, y, \theta) \in \Omega \\ 1-\frac{\alpha}{4 \pi},(r, y, \theta) \in S^{\prime}, \\ 0,(r, y, \theta) \notin\left(\Omega \cup S^{\prime}\right)\end{array}\right.$

where $\Omega$ denotes acoustic analysis domain for the wheel, $S^{\prime}$ denotes the wheel surface, and $\alpha$ denotes the three-dimensional angle of observer point $(r, y, \theta)$ to the acoustic analysis domain.

Since $\tilde{p}(r, y, \theta)$ is periodic function of $\theta$ with the period being $2 \pi$, it can be expressed as a Fourier series,

$\tilde{p}(r, y, \theta)=\sum_{m=-\infty}^{\infty} \bar{p}_{m}(r, y) \mathrm{e}^{\mathrm{i} m \theta}$.

Then, Eq. (14) becomes [13-16]

$$
\begin{gathered}
\bar{C}(r, y) \bar{p}_{m}(r, y) \\
=-\int_{\Gamma}\left[\bar{p}_{m}\left(r^{\prime}, y^{\prime}\right) \frac{\partial \bar{G}_{m}\left(r^{\prime}, y^{\prime} ; r, y\right)}{\partial n_{r^{\prime} y^{\prime}}}\right. \\
\left.\quad+\mathrm{i} \rho \omega \bar{v}_{n m}\left(r^{\prime}, y^{\prime}\right) \bar{G}_{m}\left(r^{\prime}, y^{\prime} ; r, y\right)\right] \mathrm{d} \Gamma, \\
\bar{p}_{m}\left(r^{\prime}, y^{\prime}\right)=\frac{1}{2 \pi} \int_{0}^{2 \pi} \tilde{p}\left(r^{\prime}, y^{\prime}, \theta^{\prime}\right) \mathrm{e}^{-\mathrm{i} m \theta^{\prime}} \mathrm{d} \theta^{\prime}, \\
\bar{v}_{n m}\left(r^{\prime}, y^{\prime}\right)=\frac{1}{2 \pi} \int_{0}^{2 \pi} \tilde{v}_{n}\left(r^{\prime}, y^{\prime}, \theta^{\prime}\right) \mathrm{e}^{-\mathrm{i} m \theta^{\prime}} \mathrm{d} \theta^{\prime}, \\
\bar{G}_{m}\left(r^{\prime}, y^{\prime} ; r, y\right)=\frac{r^{\prime}}{4 \pi} \int_{0}^{2 \pi} \frac{\mathrm{e}^{-\mathrm{i} k R}}{R} \mathrm{e}^{-\mathrm{i} m \theta} \mathrm{d} \theta,
\end{gathered}
$$

$$
\begin{aligned}
& \frac{\partial \bar{G}_{m}\left(r^{\prime}, y^{\prime} ; r, y\right)}{\partial n_{r^{\prime} y^{\prime}}} \\
& =-\frac{r^{\prime}}{4 \pi} \int_{0}^{2 \pi} \frac{[\mathrm{i} k+1 / R]\left[\left(y^{\prime}-y\right) n_{y^{\prime}}+\left(r^{\prime}-r \cos \theta\right) n_{r^{\prime}}\right]}{R^{2}} \\
& \quad \times \mathrm{e}^{-\mathrm{i} k R} \mathrm{e}^{-\mathrm{i} m \theta} \mathrm{d} \theta \\
& \bar{C}(r, y)=\left\{\begin{array}{l}
1,(r, y) \in S \\
1-\frac{\beta}{2 \pi},(r, y) \in \Gamma \\
0,(r, y) \notin(S \cup \Gamma)
\end{array}\right.
\end{aligned}
$$

where $R=\sqrt{\left(y^{\prime}-y\right)^{2}+r^{\prime 2}+r^{2}-2 r^{\prime} r \cos \theta}, \boldsymbol{n}_{r^{\prime} y^{\prime}}$ is the unit normal vector at the location $\left(r^{\prime}, y^{\prime}\right)$ on the cross section boundary of the wheel in the ry plane and $n_{r^{\prime}}$ and $n_{y^{\prime}}$ are directional cosines of the unit normal vector at the location $\left(r^{\prime}, y^{\prime}\right)$ on the cross section boundary of the wheel in the $r y$ plane. Equation (17) is a $2.5 \mathrm{D}$ acoustic boundary integral equation. The corresponding $2.5 \mathrm{D}$ boundary element equation can be obtained by a $1 \mathrm{D}$ mesh over the boundary of the wheel cross section containing the wheel axis with 3-noded elements. The nodal normal vibration velocity of the cross section boundary of the wheel can be obtained by using the 2.5D FEM, as shown in Sect. 2. In order to obtain the nodal sound pressures, making the point $(r, y)$ coincide with each node (allocation node) on the boundary, then the boundary element equation can be obtained

$\boldsymbol{H} \overline{\boldsymbol{p}}_{m}+\mathrm{i} \rho \omega \boldsymbol{G} \overline{\boldsymbol{v}}_{n m}=0$,

where $\boldsymbol{H}$ and $\boldsymbol{G}$ are $N \times N$ matrix and $\overline{\boldsymbol{p}}_{\boldsymbol{m}}$ is the nodal pressure vector of the cross section boundary of the wheel, respectively.

The CHIFF method is used to eliminate singularities which may occur at the characteristic frequencies of the acoustic cavity defined by the wheel surface [19]. The boundary element equation with CHIEF treatment can be written as

$$
\left[\begin{array}{c}
\boldsymbol{H} \\
\boldsymbol{H}_{\mathrm{C}}
\end{array}\right] \overline{\boldsymbol{p}}_{m}+\mathrm{i} \rho \omega\left[\begin{array}{c}
\boldsymbol{G} \\
\boldsymbol{G}_{\mathrm{C}}
\end{array}\right] \overline{\boldsymbol{v}}_{n m}=0,
$$

where $\boldsymbol{H}_{\mathrm{C}}$ and $\boldsymbol{G}_{\mathrm{C}}$ are the coefficient matrices corresponding to the CHIEF points. Now by submitting the nodal normal vibration velocity vector, $\overline{\boldsymbol{v}}_{n m}$ of the cross section boundary of the wheel into Eq. (24), the nodal sound pressure vector $\overline{\boldsymbol{p}}_{m}$ can be obtained. Following the common procedure of conventional BEM, sound pressures in the acoustic field can be predicted. 


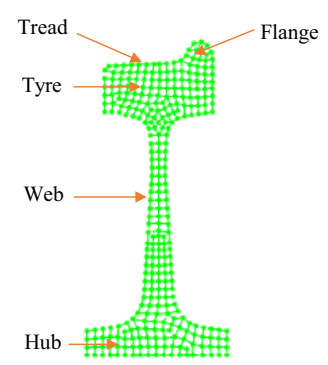

(a)

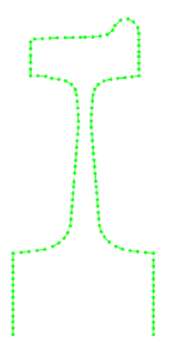

(c)

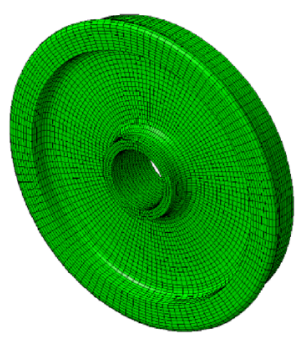

(b)

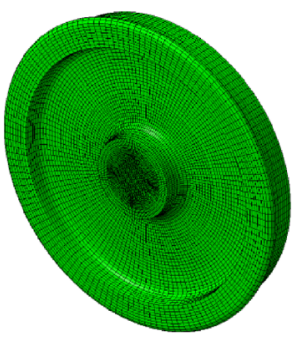

(d)

Fig. 4 Finite element and boundary element meshes of the wheel. a 2.5D FEM mesh (MATLAB), b 3D FEM mesh (ANSYS), c 2.5D BEM mesh (MATLAB) and d 3D BEM mesh (LMS Virtual.Lab)

\subsection{Sound power radiated from the wheel}

Sound power radiated from the wheel is given by the following equation

$$
\begin{aligned}
& W=\frac{1}{2} \int_{S^{\prime}} \operatorname{Re}\left[\tilde{p}\left(r^{\prime}, y^{\prime}, \theta^{\prime}\right) \tilde{v}_{n}^{*}\left(r^{\prime}, y^{\prime}, \theta^{\prime}\right)\right] \mathrm{d} S \\
& =\frac{1}{2} \int_{S^{\prime}}\left\{\operatorname{Re}\left[\tilde{p}\left(r^{\prime}, y^{\prime}, \theta^{\prime}\right)\right] \operatorname{Re}\left[\tilde{v}_{n}\left(r^{\prime}, y^{\prime}, \theta^{\prime}\right)\right]+\operatorname{Im}\left[\tilde{p}\left(r^{\prime}, y^{\prime}, \theta^{\prime}\right)\right] \operatorname{Im}\left[\tilde{v}_{n}\left(r^{\prime}, y^{\prime}, \theta^{\prime}\right)\right]\right\} \mathrm{d} S \\
& =\frac{1}{2} \int_{S^{\prime}}\left\{\begin{array}{c}
\operatorname{Re}\left[\sum_{m=-\infty}^{\infty} \bar{p}_{m}\left(r^{\prime}, y^{\prime}\right) \mathrm{e}^{\mathrm{i} m \theta^{\prime}}\right] \operatorname{Re}\left[\sum_{m^{\prime}=-\infty}^{\infty} \bar{v}_{n m^{\prime}}\left(r^{\prime}, y^{\prime}\right) \mathrm{e}^{\mathrm{i} m^{\prime} \theta^{\prime}}\right] \\
+\operatorname{Im}\left[\sum_{m=-\infty}^{\infty} \bar{p}_{m}\left(r^{\prime}, y^{\prime}\right) \mathrm{e}^{\mathrm{i} m \theta^{\prime}}\right] \operatorname{Im}\left[\sum_{m^{\prime}=-\infty}^{\infty} \bar{v}_{n m^{\prime}}\left(r^{\prime}, y^{\prime}\right) \mathrm{e}^{\mathrm{i} m^{\prime} \theta^{\prime}}\right]
\end{array}\right\} \mathrm{d} S \\
& =\pi \sum_{m=-\infty}^{\infty} \int_{\Gamma}\left\{\operatorname{Re}\left[\bar{p}_{m}\left(r^{\prime}, y^{\prime}\right)\right] \operatorname{Re}\left[\bar{v}_{n m}\left(r^{\prime}, y^{\prime}\right)\right]+\operatorname{Im}\left[\bar{p}_{m}\left(r^{\prime}, y^{\prime}\right)\right] \operatorname{Im}\left[\bar{v}_{n m}\left(r^{\prime}, y^{\prime}\right)\right]\right\} r \mathrm{~d} \Gamma .
\end{aligned}
$$

Equation (25) shows that the total sound power is the sum of sound powers at each circumferential order. Integrals in Eq. (25) are evaluated using the boundary element method combined with the nodal normal vibration velocity vector $\overline{\boldsymbol{v}}_{n m}$ and nodal pressure vector $\overline{\boldsymbol{p}}_{m}$ of the cross section boundary of the wheel.

\section{Results}

Results are produced for a wheel used in a trailer coach of a Chinese high-speed train. The rolling radius of the wheel is $0.46 \mathrm{~m}$. Material parameters are density $7850 \mathrm{~kg} / \mathrm{m}^{3}$, Young's modulus $210 \mathrm{GPa}$, Poisson ratio 0.30 , and loss factor 0.005 . The fluid medium is air at normal conditions, with density $1.21 \mathrm{~kg} / \mathrm{m}^{3}$ and sound speed $344 \mathrm{~m} / \mathrm{s}$. According to the 2.5D FEM and 2.5D BEM, only the cross section of the wheel has to be meshed. The finite element and boundary element meshes of the cross section are shown in Fig. 4.

For comparison, 3D analyses are performed using commercial software ANSYS for vibration and LMS Virtual.Lab for sound radiation. The corresponding 3D meshes are also shown in Fig. 4.

It is known that the sound pressure spectrum due to a unit harmonic wheel-rail force multiplied by the actual wheel-rail force spectrum gives the actual sound pressure spectrum, and the sound power spectrum due to a unit harmonic wheel-rail force multiplied by the squared magnitude of actual wheel-rail force spectrum produces the actual sound power spectrum. Thus, to meet the purpose of this work, it is reasonable to consider a wheel 
subject to a unit vertical harmonic wheel-rail force at the wheel-rail contact point.

In the calculation of the vibration characteristics of the wheel, the axle is not taken into account in the model, and RBE3 elements (see ANSYS manual) are used to make the wheel axis move vertically only. Considered frequency range is $10-5000 \mathrm{~Hz}$, and the frequency resolution is $10 \mathrm{~Hz}$. In the calculation of the sound radiation characteristics of the wheel, the free field condition is assumed, and elements are added to prevent sound leakage through the hub hole. The frequency range investigated is $20-4000 \mathrm{~Hz}$ with a resolution of $10 \mathrm{~Hz}$.

\subsection{Vibration characteristic analysis}

\subsubsection{Comparison in wheel vibration between 2.5D FEM and $3 D$ FEM}

The comparison in the vertical receptance of the wheel not in rotation at the wheel-rail contact point between 2.5D FEM (programmed in MATLAB) and 3D FEM (ANSYS) is shown in Fig. 5. It can be seen from Fig. 5 that the amplitude, real and imaginary parts of the receptance calculated by the two methods are in good agreement, so the derivation of the 2.5D FEM and the related program are correct. It can be seen that resonances occur to the receptance for frequency higher than $1500 \mathrm{~Hz}$. The first peak at $1590 \mathrm{~Hz}$ corresponds to a mode which exhibits 2 nodal diameters, and the second peak at $2400 \mathrm{~Hz}$ corresponds to a mode having 3 nodal diameters. These two modes mainly behave as stretching vibrations along the radial, and they are the main modes that affect the tread vibration.

\subsubsection{Influence of rotation speed on wheel vibration}

The influence of wheel rotation speed on the vertical receptance of the wheel at the wheel-rail contact point is shown in Fig. 6. Investigated train speeds are 0, 200, 300, and $400 \mathrm{~km} / \mathrm{h}$, or equivalently, $0,1153,1730$, and $2306 \mathrm{rpm}$. Here a 'zero train speed' is used just to indicate that wheel rotation is not considered in the analysis, rather than that the train is not running.

Figure 6 shows that the wheel rotation speed splits most of the peaks of the receptance into two lower peaks. The higher the wheel rotation speed and the frequency are, the greater the splitting of the peak. This is the combined result of the gyroscopic effect of the wheel rotation and the rotating of the wheel-rail force along the periphery of the wheel [12]. According to Ref. [12], the latter plays a more important role than the former for peak splitting.

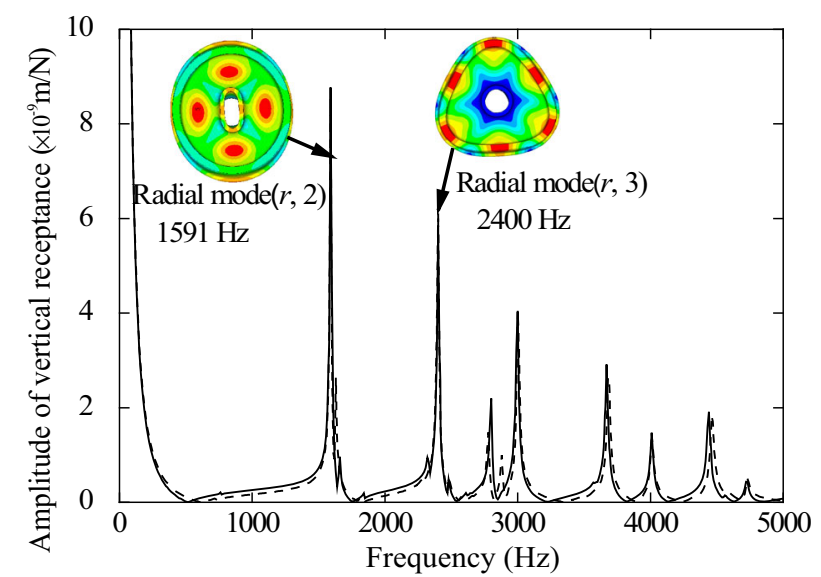

(a)

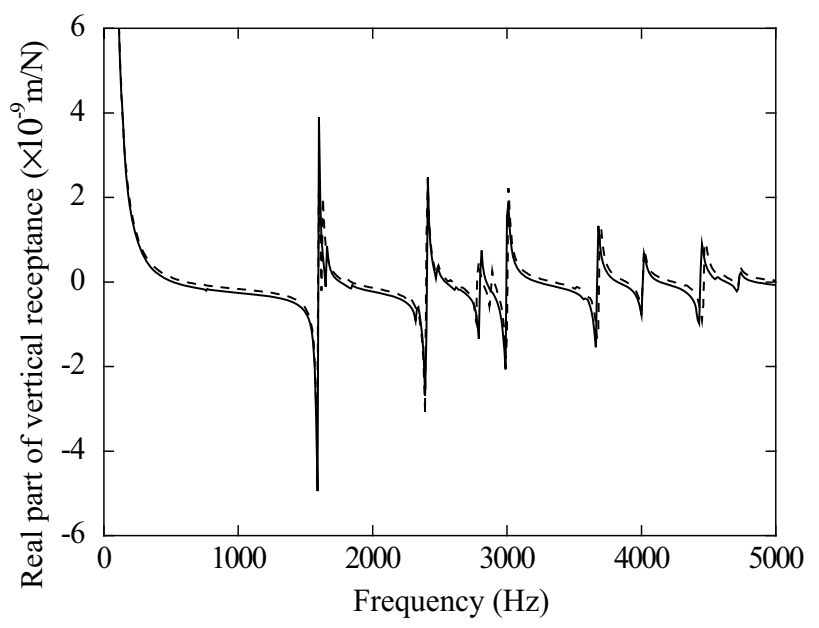

(b)

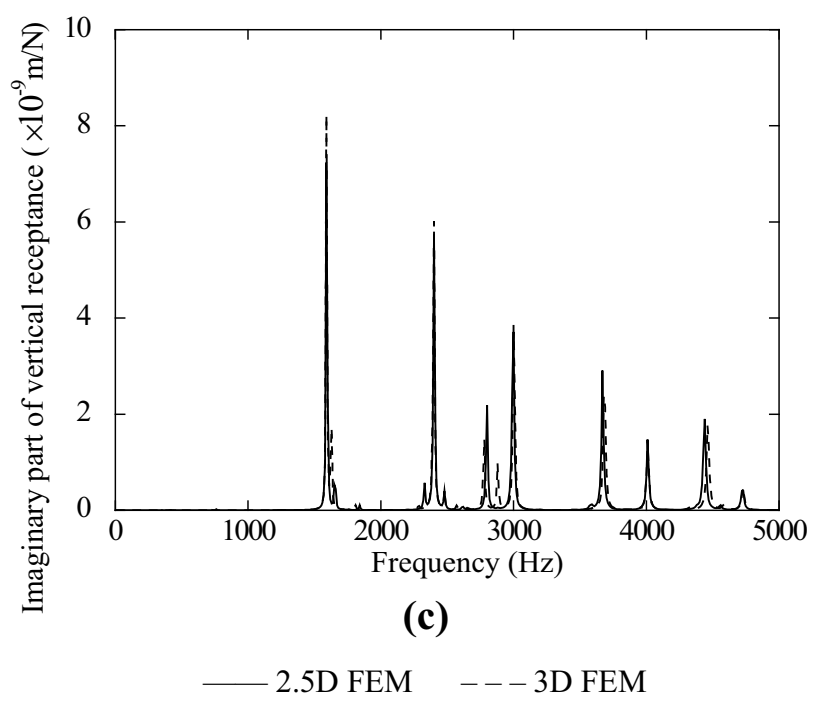

Fig. 5 Comparison in the vertical receptance of the wheel at the wheel-rail contact point. a Amplitude, $\mathbf{b}$ real part, $\mathbf{c}$ imaginary part 
Fig. 6 Influence of the rotation speed on the wheel vertical receptance at the wheel-rail contact point. a 0 and $200 \mathrm{~km} / \mathrm{h}$, b 0 and $300 \mathrm{~km} / \mathrm{h}$ and $\mathbf{c} 0$ and $400 \mathrm{~km} / \mathrm{h}$

\subsection{Sound radiation characteristic analysis}

\subsubsection{Comparison in wheel sound radiation between ' $2.5 D$ $F E M+2.5 D$ BEM' and ' $2.5 D F E M+3 D B E M$ '}

4.2.1.1 Comparison in sound pressure level Sound pressures at two standard measurement points are used to compare the methods. These two standard measurement points are defined according to Railway applicationsAcoustics-Measurement of noise emitted by railbound vehicle (ISO 3095: 2005). They are away from the track central line with a lateral distance of $7.5 \mathrm{~m}$, but one (point A) is $1.2 \mathrm{~m}$ above the top surface of rail, and the other (point B) is $3.5 \mathrm{~m}$ above. See Fig. 7.

The comparisons between '2.5D FEM + 2.5D BEM' and '2.5D FEM + 3D BEM' are shown in Fig. 8 for the wheel at stationary. The normal vibration velocity of the wheel calculated by the 2.5D FEM is taken as the acoustic boundary condition for both of the methods. It can be seen from Fig. 8 that the sound pressure levels at the standard measuring points calculated by the two methods are in good agreement, especially at resonance frequencies. However, differences are present around frequencies at which pressure level dips and the cause is yet to be determined.

4.2.1.2 Comparison in sound power level The comparison in radiated sound power level between '2.5D FEM + 2.5D BEM' and '2.5D FEM + 3D BEM' is shown in Fig. 9. It can be seen from Fig. 9 that agreement in radiated sound power level between these two methods is even better, further validating the 2.5D BEM and related programs. It is also seen that, for a given wheel-rail force magnitude, the wheel generates more noise when the force frequency is higher than $1500 \mathrm{~Hz}$. The comparison between Figs. 5 and 9 shows that, for frequencies less than $1000 \mathrm{~Hz}$, three resonances occur at the wheel to radiate sound but these resonances do not appear in the vertical receptance of the wheel at the wheel/rail contact point.

It can also be concluded through these comparisons that the '2.5D FEM + 2.5D BEM' is much more computationally efficient. In fact, based on the rule that at least 6 elements should be used per wavelength, the 3D BEM model is too large to perform sound radiation prediction for frequencies than $4000 \mathrm{~Hz}$ on a Dell Precision Tower 7910 workstation, while the 2.5D BEM can be run on a laptop.

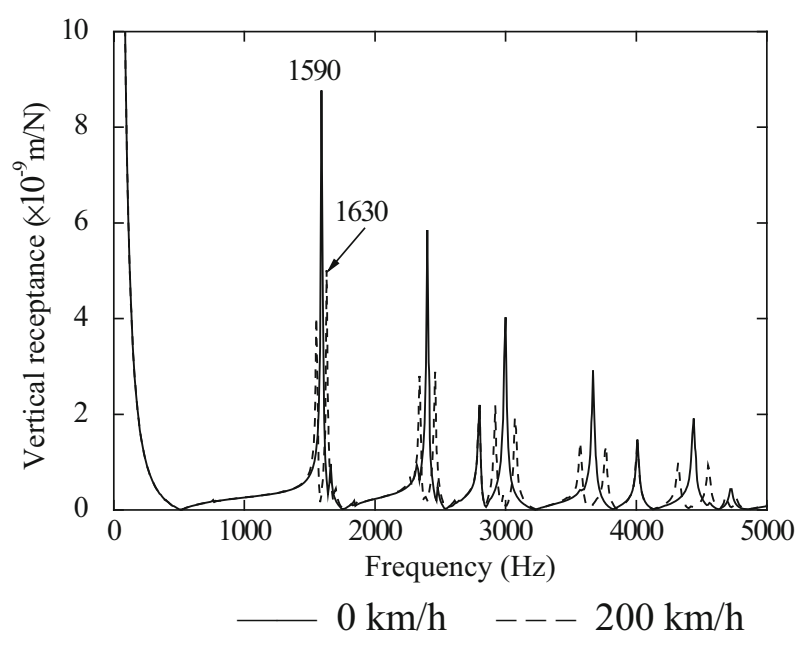

(a)

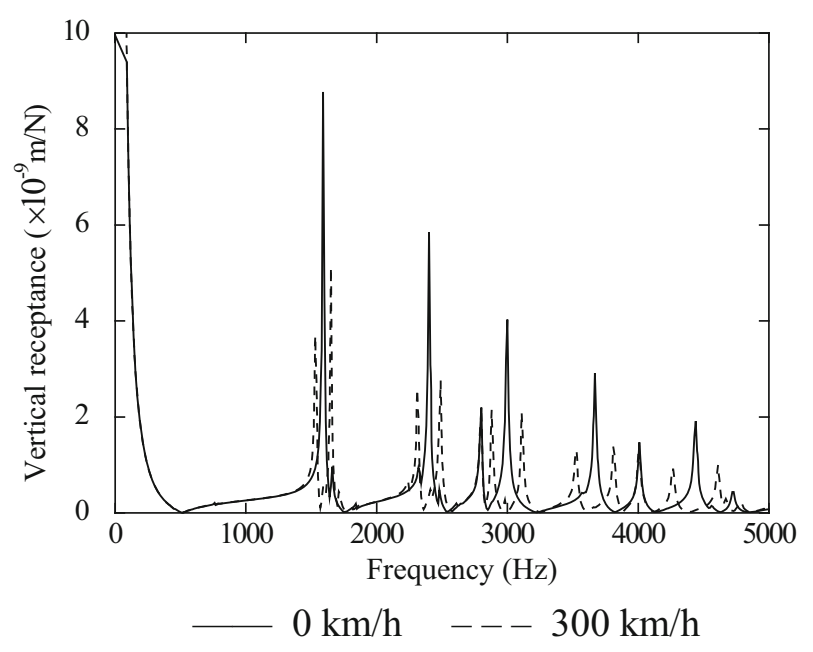

(b)

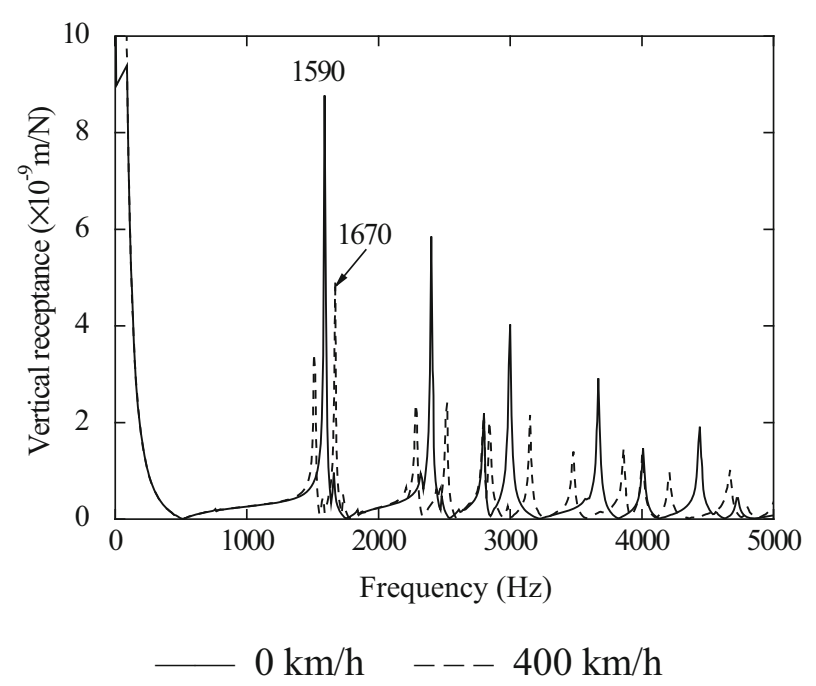

(c) 


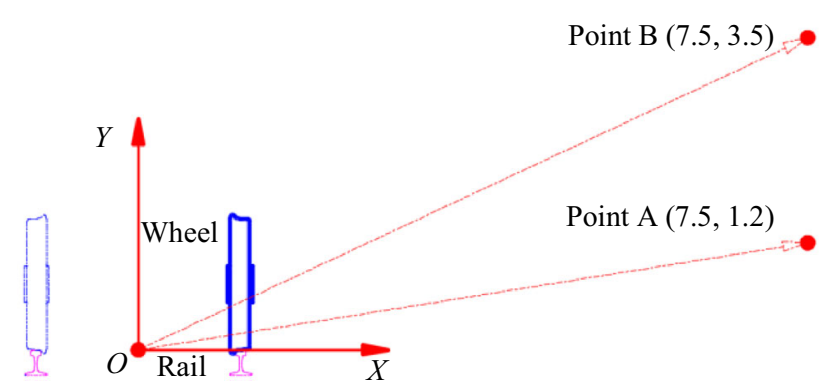

Fig. 7 Standard measurement points

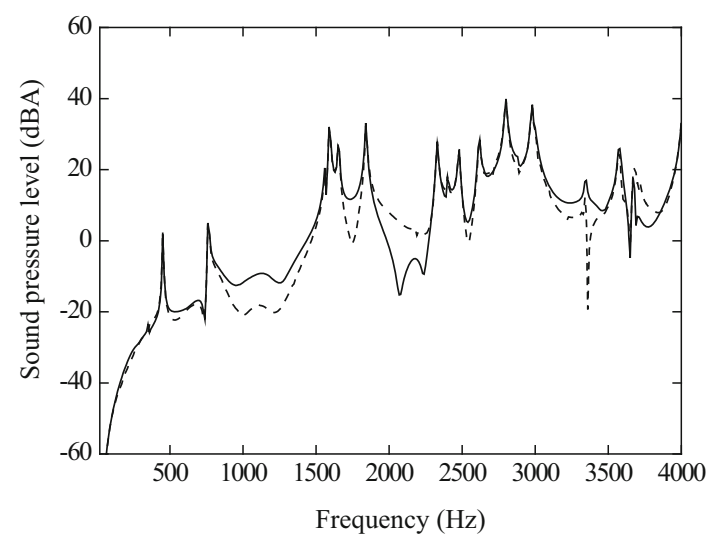

(a)

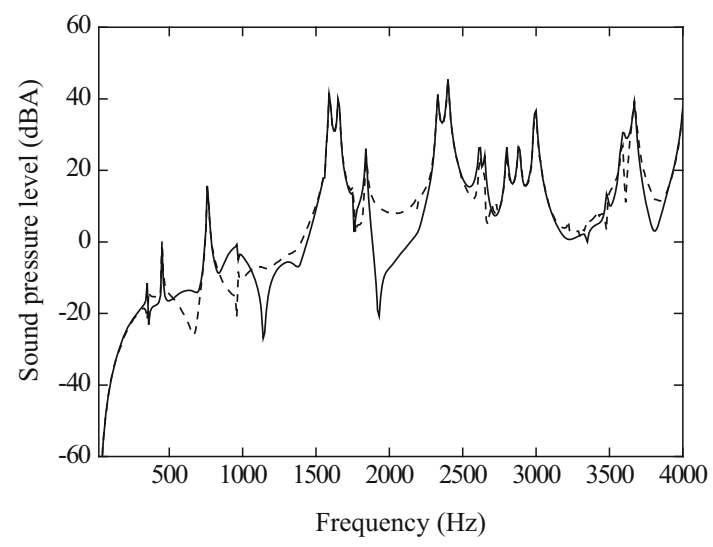

(b)

$-2.5 \mathrm{D}$ FEM $+2.5 \mathrm{D}$ BEM $\quad---2.5 \mathrm{D}$ FEM+3D BEM

Fig. 8 Comparisons of the sound pressure levels at the standard measured points. a Standard measuring point $\mathrm{A}$ and $\mathbf{b}$ standard measuring point $\mathrm{B}$

\subsubsection{Influence of the rotation speed on wheel sound radiation}

4.2.2.1 Influence on sound pressure level at the standard measuring point $A$ The influence of wheel rotation speed on the sound pressure level at the standard measuring point A is shown in Fig. 10 and that on the radiated sound power is shown in Fig. 11. Again, the investigated train speeds are $0,200,300$ and $400 \mathrm{~km} / \mathrm{h}$, and the wheel is subject to a unit vertical harmonic wheel-rail force at the wheel-rail contact point.

It can be seen from Fig. 10 that the wheel rotation speed splits most peaks into two peaks, and the higher the wheel rotation speed and the frequency are, the greater the splitting of the peak is. This is more vividly shown in the colour map.

The splitting of a peak in the sound pressure level spectrum at a position may cause difficulties to interpret measurement results. For example, due to the splitting, a peak in sound pressure measured near a wheel cannot straightforwardly be associated with a normal mode of the wheel, or with wheel/rail roughness at a particular wavelength.

4.2.2.2 Influence on radiated sound power level The effect of wheel rotation speed on sound power (Fig. 11) is similar to that on sound pressure, i.e. the wheel rotation speed splits most peaks into two peaks, and the higher the wheel rotation speed and the frequency are, the greater the splitting of the peak is. The colour map shows a more vivid picture.

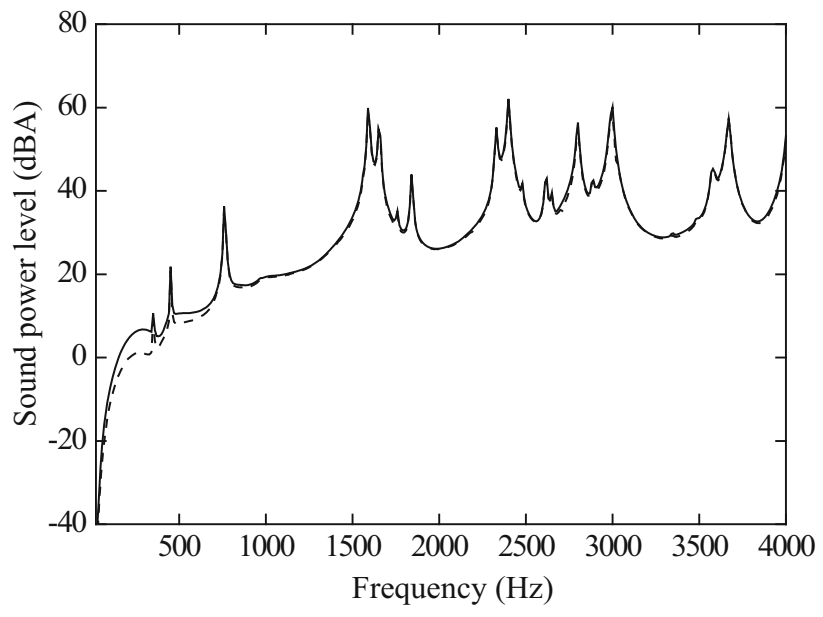

2.5D FEM+2.5D BEM - - 2.5D FEM+3D BEM

Fig. 9 Comparison of the radiated sound power levels 


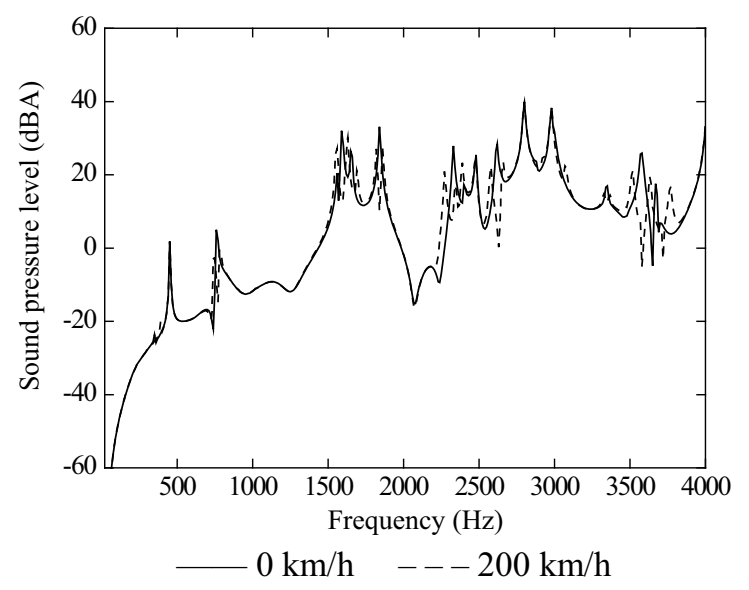

(a)

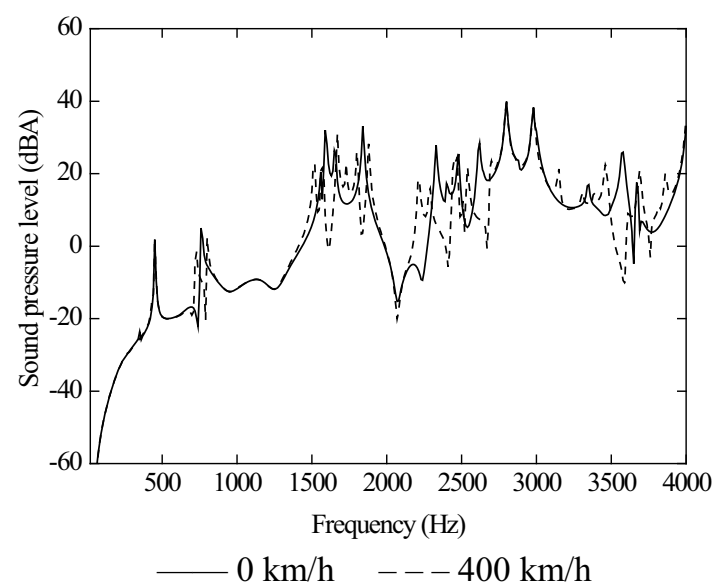

(c)

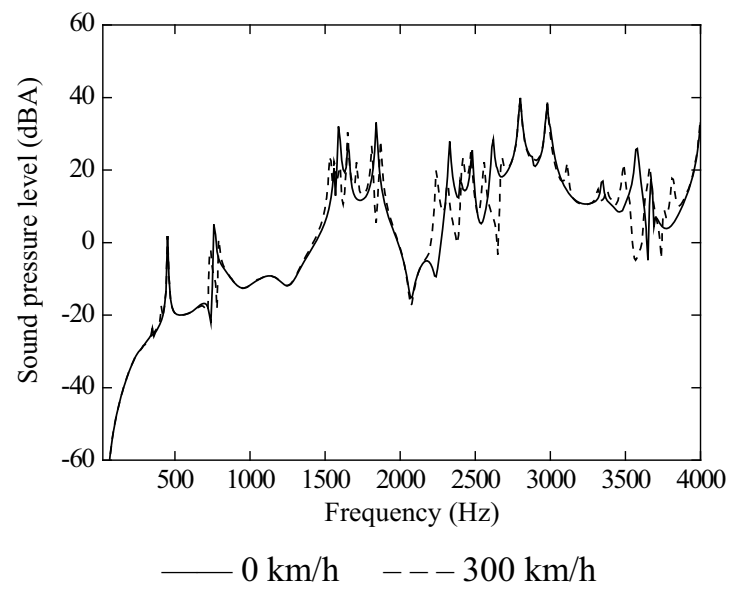

(b)

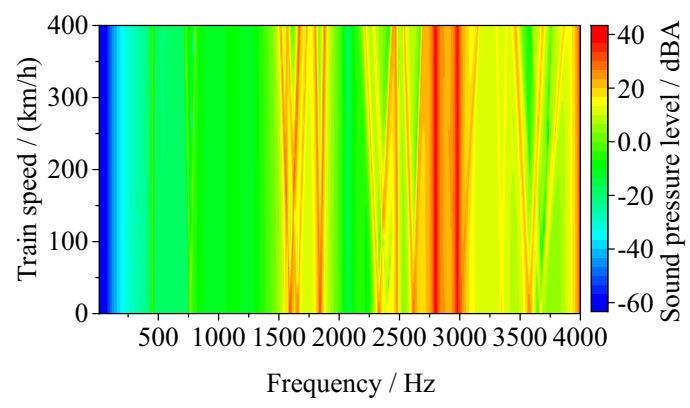

(d)

Fig. 10 Influences of the rotation speed on the sound pressure level at the standard measuring point A. a 0 and $200 \mathrm{~km} / \mathrm{h}, \mathbf{b} 0$ and $300 \mathrm{~km} / \mathrm{h}, \mathbf{c} 0$ and $400 \mathrm{~km} / \mathrm{h}$ and d $0-400 \mathrm{~km} / \mathrm{h}$

4.2.2.3 Influence on sound radiation directivity The directivity of sound radiation is described by sound pressure levels on a sphere which is large enough to surround the radiator at the sphere centre. In practice, the measurement point of the sound radiation of the wheel needs to be made in some location in the sound field, so it is important to understand the spatial distribution of the radiated sound field and the influence of the rotating speed on the wheel directivity. For better visualisation, this paper only presents sound pressure levels along a 2-m-radius circle concentric with the nominal rolling circle of the wheel, and ones along a horizontal circle of the same radius containing the wheel axis.

The influences of the wheel rotation speed on radiation directivity are shown in Fig. 12 for three frequencies, $1590 \mathrm{~Hz}$ (Fig. 12a, b), $1630 \mathrm{~Hz}$ (Fig. 12c, d) and $1670 \mathrm{~Hz}$
(Fig. 12e, f). The first frequency is the one at which the vertical wheel receptance of the wheel not in rotation reaches the maximum (Fig. 6a). This peak will be split into two peaks if the wheel is in rotation, as shown in Fig. 6b, c. The second and third frequencies are associated with the upper peak for the wheel rotating at 200 and $400 \mathrm{~km} / \mathrm{h}$, respectively.

It can be seen from Fig. 12 that: (1) the radiated sound field of the wheel is not a simple monopole sound field; lobes are presented in directivity; (2) for a given frequency, rotation speed changes directivity; (3) for a given frequency and a given rotation speed, the vertical and horizontal directivities are quite similar; (4) directivity for the frequency at which the upper peak occurs does not change with rotation speed significantly. 
4.2.2.4 On contributions of different parts of the wheel to total sound power The influences of the wheel rotation speed on the radiation power contributions (the ratio of sound power radiated by a part to the total radiated sound power of the wheel) of the hub, web and tyre (including tyre, tread and flange) are shown in Fig. 13. It can be seen from Fig. 13 that: (1) the web is the most important radiator, followed by the tyre; (2) compared to that from the other two, sound radiation from the hub is negligible; (3) the wheel rotation speed has a significant effect on the peak values of the sound power contribution of the web, or equivalently, on the trough values of the sound power contribution of the tyre. In other words, rotation speed has more effect on sound radiation from the web.

To sum up, it is necessary to consider the rotation of the wheel when calculating the sound radiation of the wheel.

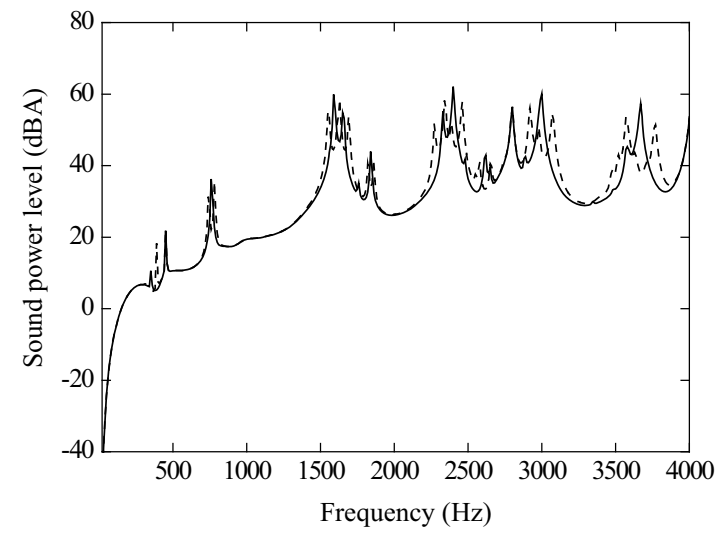

$0 \mathrm{~km} / \mathrm{h} \quad---200 \mathrm{~km} / \mathrm{h}$

(a)

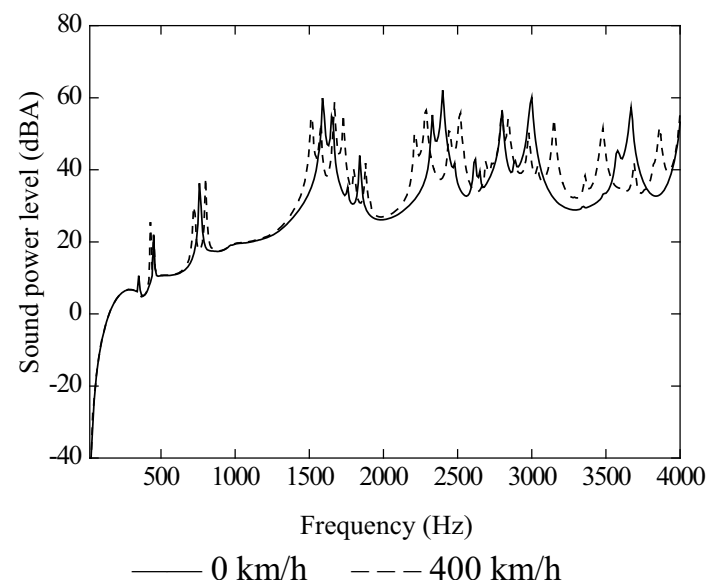

(c)
Fig. 12 Influences of the rotation speed on the directivity corresponding to the typical peak frequencies. a Vertical directivity at $1590 \mathrm{~Hz}$, b horizontal directivity at $1590 \mathrm{~Hz}$, c vertical directivity at $1630 \mathrm{~Hz}, \mathbf{d}$ horizontal directivity at $1630 \mathrm{~Hz}$, e vertical directivity at $1670 \mathrm{~Hz}$ and $\mathbf{f}$ horizontal directivity at $1670 \mathrm{~Hz}$

\section{Conclusions}

In this paper, by making use of the axial symmetry of the wheel, 2.5D FEM and 2.5D BEM are combined to calculate the vibration and sound radiation of a train wheel subject to a unit vertical harmonic wheel-rail force. Then, the 2.5D FEM and relevant programs are validated by comparing results from this procedure with those from conventional 3D analyses using ANSYS, and the 2.5D BEM and relevant programs are validated by comparing results from this procedure with those from conventional

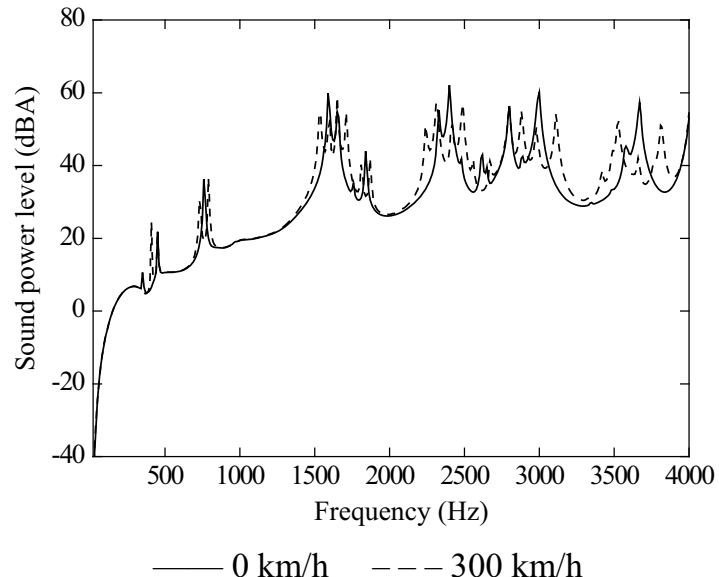

(b)

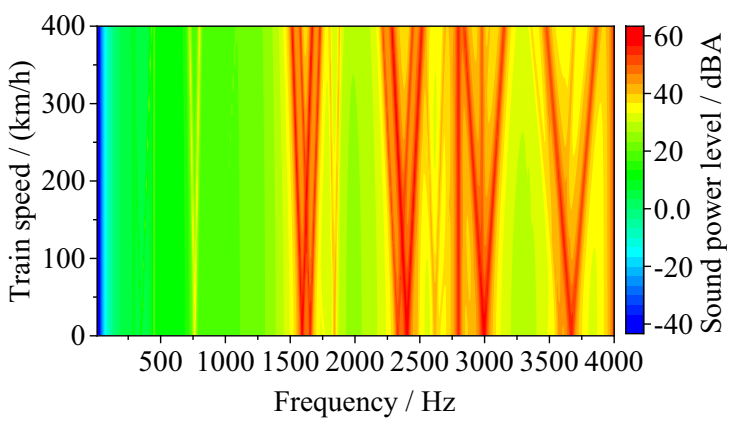

(d)

Fig. 11 Influences of the rotation speed on the sound power level of the wheel. a 0 and $200 \mathrm{~km} / \mathrm{h}, \mathbf{b} 0$ and $300 \mathrm{~km} / \mathrm{h}, \mathbf{c ~} 0$ and $400 \mathrm{~km} / \mathrm{h}$ and d $0-400 \mathrm{~km} / \mathrm{h}$ 


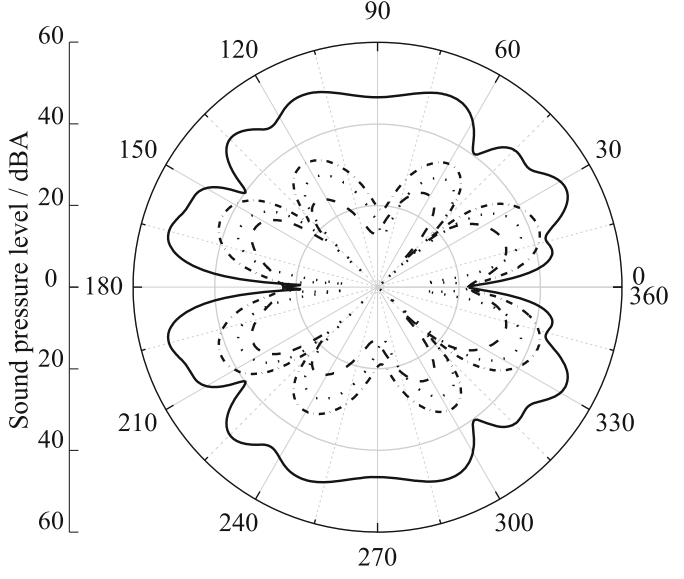

(a)

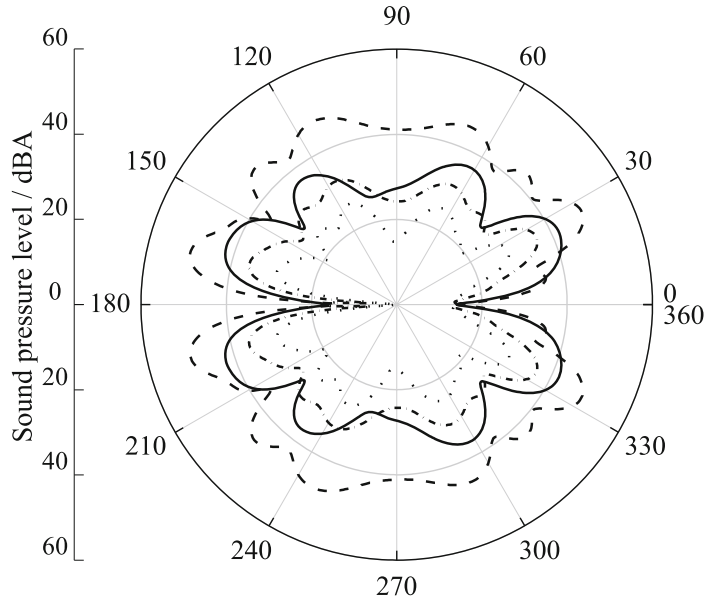

(c)

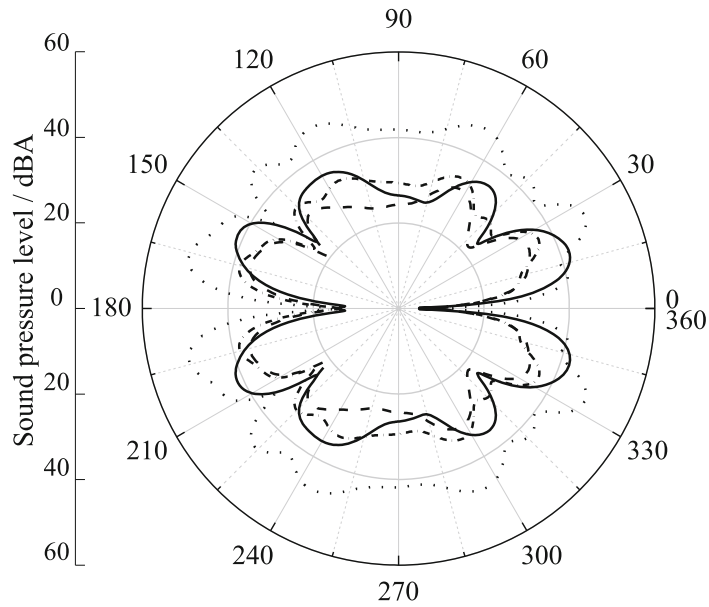

(e)

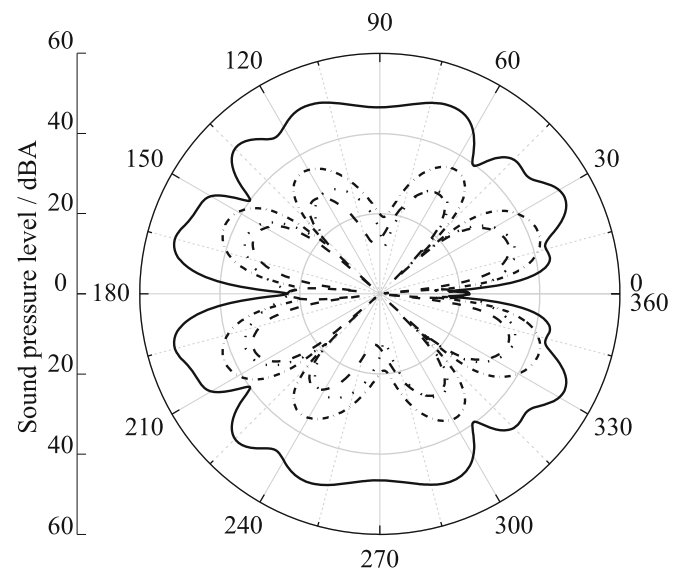

(b)

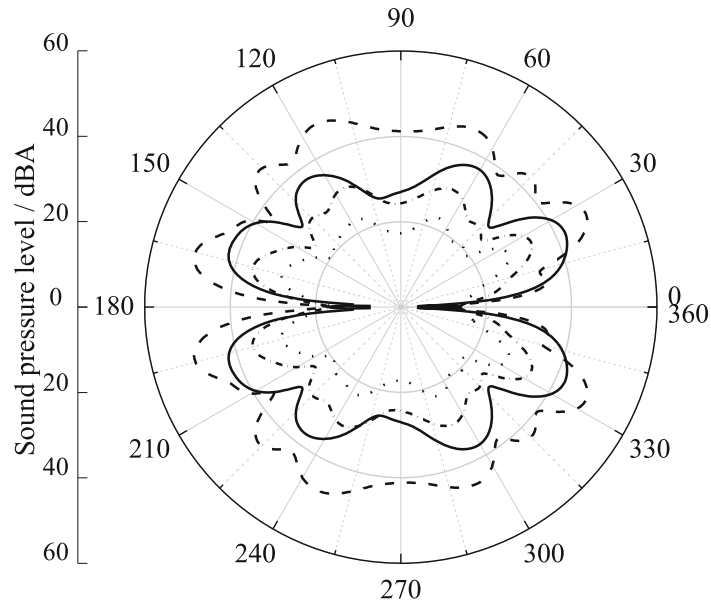

(d)

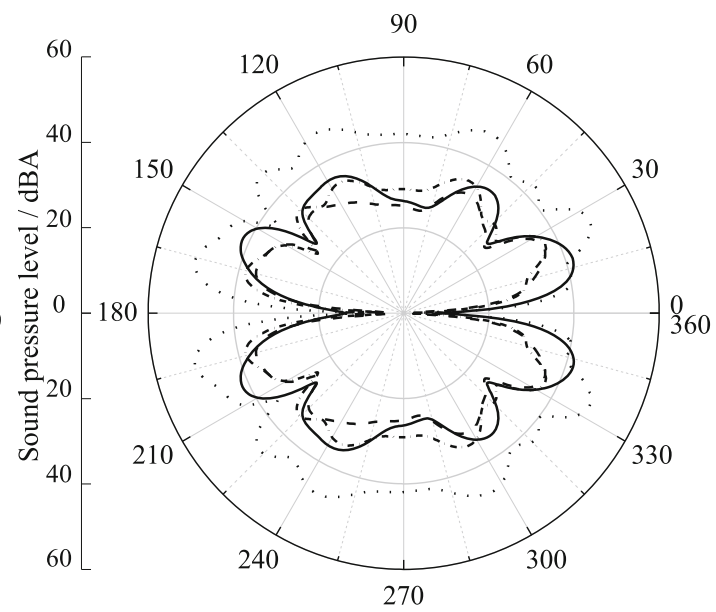

(f)

$0 \mathrm{~km} / \mathrm{h} \quad---200 \mathrm{~km} / \mathrm{h} \quad-\cdot-300 \mathrm{~km} / \mathrm{h}$

$400 \mathrm{~km} / \mathrm{h}$ 


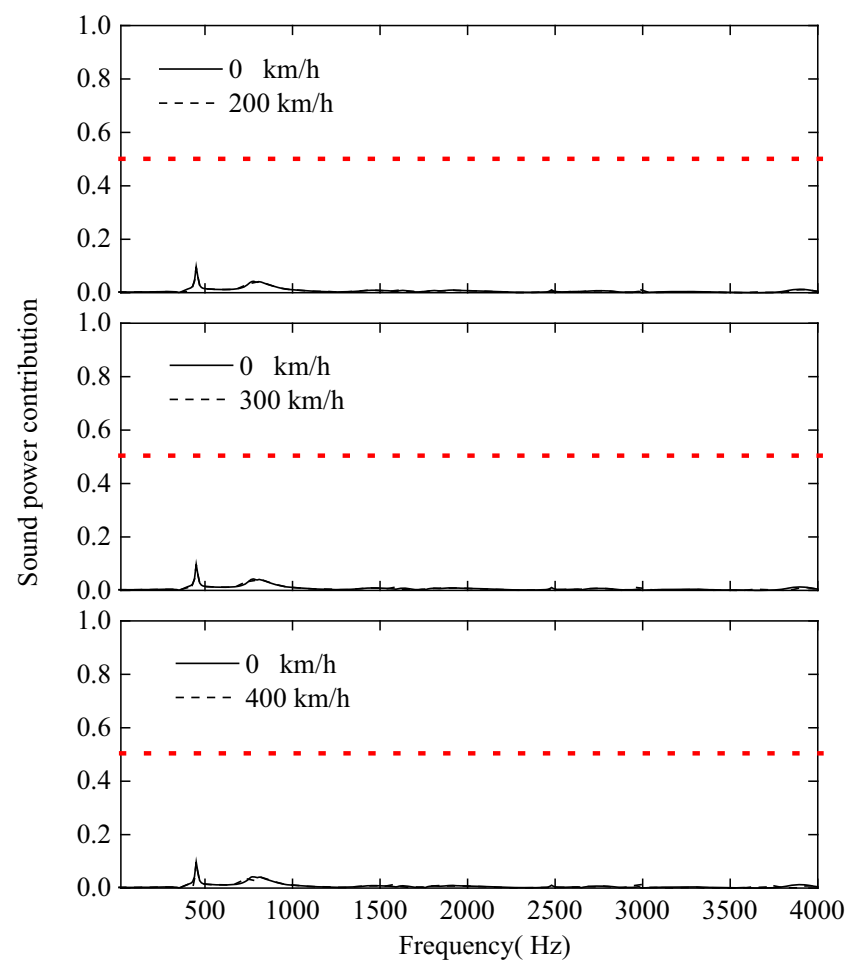

(a)

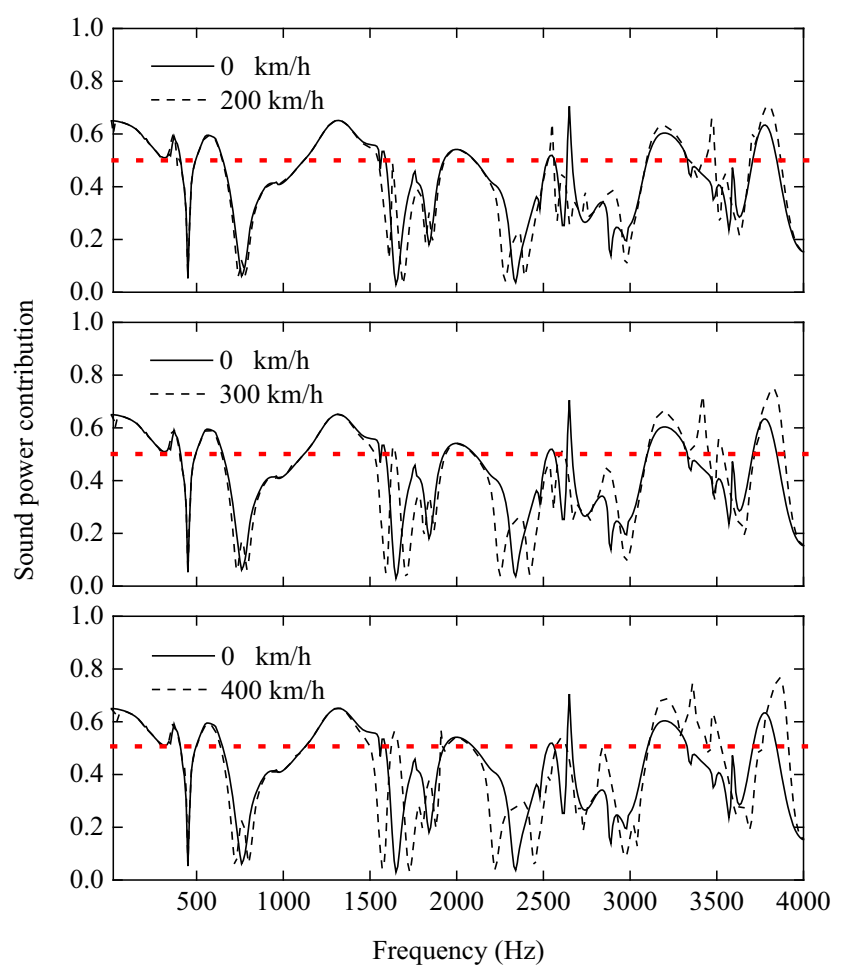

(b)

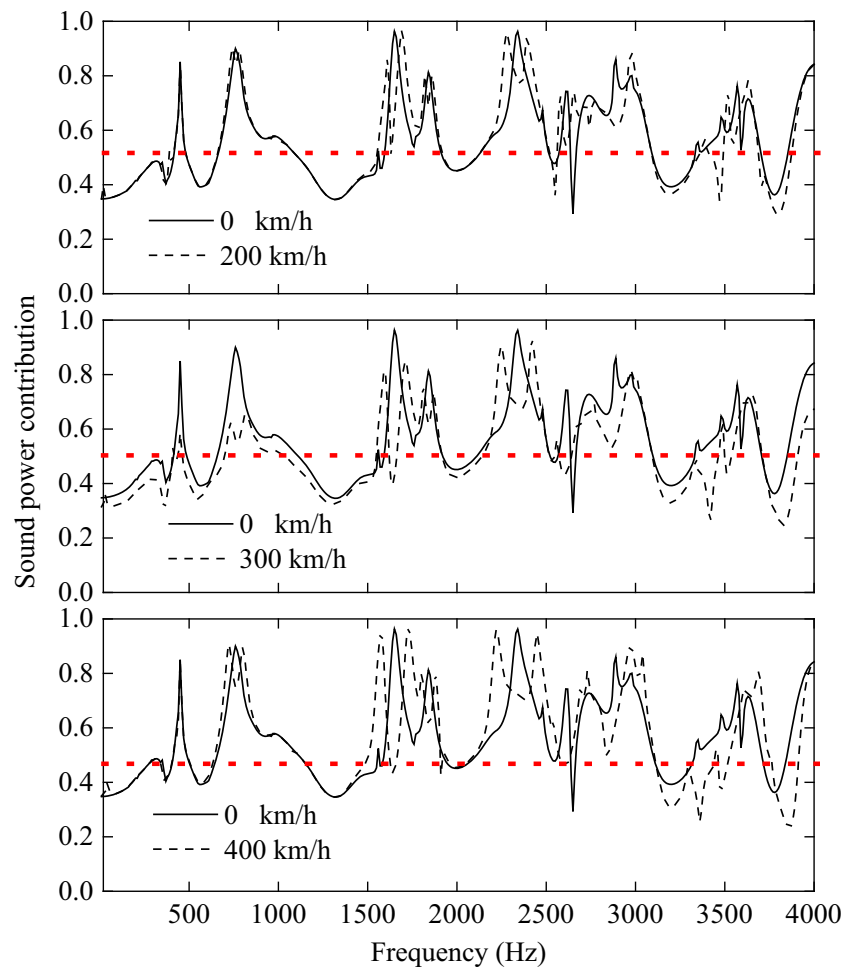

(c)

Fig. 13 Influence of the rotation speed on the radiation power contributions of each part of the wheel. a Hub, b web, c tyre 
3D analyses using LMS Virtual.Lab. Finally, the influences of the wheel rotation speed on the vibration response and sound radiation of the wheel are studied.

1. The wheel rotation speed splits most peaks of the vertical receptance at the wheel-rail contact point, the sound pressure levels at the standard measuring point and the sound power level into two peaks, and the bigger the wheel rotation speed and the frequency are, the greater the splitting of the peak is;

2. The wheel rotation speed changes the vertical and horizontal directivity for a given frequency, and the vertical and horizontal directivities are quite similar for a given frequency and a given rotation speed.

3. The wheel rotation speed has a significant effect on the peak values of the sound power contribution of the web, or equivalently, on the trough values of the sound power contribution of the tyre;

4. It is necessary to consider the rotation of the wheel when calculating the vibration response and sound radiation of the wheel.

Acknowledgements The authors acknowledge the support to this work from the Ministry of Science and Technology of China (2016YFE0205200), China Railway (2015Z003-B) and Scientific Research Foundation of State Key Laboratory of Traction Power (2017TPL_T01).

\section{Compliance with ethical standards}

Conflict of interest The authors of this paper certify that they have no affiliation with, or involvement in, any organisation or entity with any financial interest, or nonfinancial interest in the subject matter or materials discussed in this manuscript.

Open Access This article is distributed under the terms of the Creative Commons Attribution 4.0 International License (http:// creativecommons.org/licenses/by/4.0/), which permits unrestricted use, distribution, and reproduction in any medium, provided you give appropriate credit to the original author(s) and the source, provide a link to the Creative Commons license, and indicate if changes were made.

\section{References}

1. Thompson DJ (2000) A review of the modelling of wheel/rail noise generation. J Sound Vib 231(3):519-536
2. Thompson DJ (1988) Predictions of acoustic radiation from vibration wheels and rails. J Sound Vib 120:275-280

3. Remington PJ (1976) Wheel/rail noise, part I: characterization of the wheel/rail dynamic system. J Sound Vib 46:359-379

4. Remington PJ (1976) Wheel/rail noise, part IV: rolling noise. J Sound Vib 46:419-436

5. Remington PJ (1987) Wheel/rail rolling noise I: theoretical analysis. J Acoust Soc Am 81(6):1805-1823

6. Remington PJ (1987) Wheel/rail rolling noise II: validation of the theory. J Acoust Soc Am 81(6):1824-1832

7. Schneider E, Popp K, Irretier H (1986) Noise generation in railway wheels due to rail-wheel contact force. J Sound Vib 120:227-244

8. Thompson DJ (1993) wheel-rail noise generation, part I, II, II, IV and V. J Sound Vib 161(3):387-482

9. Thompson DJ, Jones CJC (2002) Sound radiation from a vibrating railway wheel. J Sound Vib 253(2):401-419

10. Sato K, Sasakura M, Akutsu K et al (2006) Acoustic characteristics of wheels with different Web shapes. Q Rep Railw Tech Res Inst 47(1):28-33

11. Fang J, Xiao X, Jin X et al (2010) Effect of train speed on acoustic radiation characteristics of high-speed train wheel vibration. J Mech Eng 46(22):96-104 (in Chinese)

12. Sheng X, Liu Y, Zhou X (2016) The response of a high-speed train wheel to a harmonic wheel-rail force. J Phys Conf Ser 744:012145

13. Seybert AF, Soenarko B, Rizzo FJ et al (1986) A special integral equation formulation for acoustic radiation and scattering for axisymmetric bodies and boundary conditions. J Acoust Soc Am 80(4):1241-1247

14. Soenarko B (1993) A boundary element formulation for radiation of acoustic waves from axisymmetric bodies with arbitrary boundary conditions. J Acoust Soc Am 93(2):631-639

15. Kuijpers AHWM, Verbeek G, Verheij JW (1997) An improved acoustic Fourier boundary element method formulation using fast Fourier transform integration. $\mathrm{J}$ Acoust Soc Am 102(3):1394-1401

16. Sheng $X$ (2008) Applying the boundary element and modal superposition methods to evaluate inlet noise of high circumferential orders [M]. In: 08' International conference on noise and vibration engineering, pp 535-547

17. Wu TW (2000) Boundary element acoustics, fundamentals and computer codes. WIT Press, Southampton

18. Ji Z (2010) Acoustic theory and design of mufflers. Science Press, Beijing, pp 188-206 (in Chinese)

19. Schenck HA (1968) Improved integral formulation for acoustic radiation problems. J Acoust Soc Am 44(1):41-58 